\title{
ASSISTÊNCIA DA ENFERMAGEM NA SAÚDE DO HOMEM: UMA REVISÃO INTEGRATIVA
}

\section{NURSING CARE IN MAN'S HEALTH: AN INTEGRATIVE REVIEW}

\section{Arilton Cabral Gobira;}

Bacharel em Enfermagem. Alfa - Faculdade de Almenara, Almenara, Minas Gerais, Brasil.

\section{Clara Soares da Silva}

Bacharel em Enfermagem. Alfa - Faculdade de Almenara, Almenara, Minas Gerais,

Brasil.

Viviane Amaral Toledo Coelho

Doutora. Autor parar correspondência; vivianeatc@yahoo.com.br. Rua Rodrigues Seabra, 243, Alfa - Faculdade de Almenara, Almenara, Minas Gerais, Brasil.

Ednardo de Souza Nascimento Mestre. Alfa - Faculdade de Almenara, Almenara, Minas Gerais, Brasil. Creonice Santos Bigatello Especialista. Alfa - Faculdade de Almenara, Almenara, Minas Gerais, Brasil.

\section{RESUMO}

Assistência da Enfermagem em relação à saúde do Homem é baseada em desafios, em virtude que os homens na maioria das vezes têm dificuldades em reconhecer suas necessidades de saúde, principalmente quando estão relacionadas à prevenção. Sendo assim, esta pesquisa teve como objetivo identificar na literatura através de uma revisão integrativa a assistência de enfermagem frente a saúde do homem com foco na execução da Política Nacional de Atenção Integral à Saúde do Homem. Os dados coletados e analisados foram referentes ao período de 2014 a 2018, embasados em sites científicos 
como: Biblioteca Virtual em Saúde (BVS), Literatura Latino-Americana e do Caribe em Ciências da Saúde (LILACS), Base de Dados da Enfermagem (BDENF). No montante de 50 trabalhos acadêmicos estudados, foram escrutinados 15 artigos para a primeira seleção de trabalhos da revisão integrativa. Sendo que 10 destes foram selecionados para compor o arcabouço teórico de onde serão analisados os resultados e discussão. O estudo enfatiza a importância da enfermagem como elemento essencial na assistência, entre suas atribuições, destacam-se as ações de promoção da saúde voltada para população masculina, e que estejam em harmonia com a PNAISH.

Palavras-chaves: Assistência de Enfermagem. Atenção Primária à Saúde. Masculinidade.

Política de Saúde. Saúde do Homem.

\section{ABSTRACT}

Nursing care in relation to man health is based on challenges, since men most often have difficulties in recognizing their health needs, especially that related to prevention. Thus, this research aimed to identify in the literature, through an integrative review, the nursing assistance to the health of the man focused on the implementation of the National Policy of Integral Attention to Human Health. The data collected and analyzed is related to the period from 2014 to 2018 , based on scientific websites such as: Virtual Health Library (BVS in portuguese language), Latin American and Caribbean Literature in Health Sciences (LILACS), Nursing Database (BDENF). Among 50 academic papers studied, 15 articles were scrutinized for the first selection of papers for the integrative review. Finally 10 of these were selected to compose the theoretical framework from where the results and discussion will be analyzed. The study emphasizes the importance of nursing as an essential element in the care, among its attributions, stand out the actions of health promotion aimed at the male population, and that are in harmony with the National Policy for Comprehensive Health Care for Man (PNAISH). 
Keywords: Nursingcare. Primary Health Care. Masculinity. Health Policy. Men Health.

\section{INTRODUÇÃO}

Os homens na maioria das vezes têm dificuldade em reconhecer suas necessidades de saúde, principalmente quando estão relacionadas à prevenção. Visando isso, foi criada a Política Nacional de Atenção Integral a Saúde do Homem (PNAISH), lançada em 2009 pelo Ministério da Saúde, com o objetivo de promoção de ações de saúde que possibilitem a apreensão da realidade dos homens entre 20 e 59 anos de idade nos seus diversos contextos (BRASIL, 2009).

Segundo a PNAISH (2009), a não procura dos homens pelos serviços de saúde sinaliza a inadequação entre as necessidades e/ou expectativas de saúde dos homens e a falta de conhecimento específico por parte das equipes de saúde sobre as peculiaridades deste segmento da sociedade, particularmente na Atenção Básica (AB) à saúde.

A política de saúde do homem é parceira da política nacional de atenção primária da saúde. Por mais que tenham essa parceria, existem diversos fatores que influenciam na dificuldade dos homens em comparecer à unidade básica. De acordo com a PNAISH de 2009, isso se dá pelas barreiras socioculturais, estereótipos de gênero, medo que descubra doenças, além de barreiras institucionais, estratégias de comunicação que não privilegiam os homens, inadequação dos serviços de saúde, incompatibilidade de horários de funcionamento com o trabalho e a falta de estratégias dos profissionais da saúde para realizar uma assistência aos homens.

É perceptível uma demanda maior de mulheres que comparecem na unidade básica comparando-se aos números correspondentes ao sexo masculino. Diante disso, fica notório que a não busca dos homens por ajuda nos serviços de saúde, ocasiona o agravamento de doenças que poderiam ser alvo de ações resolutivas em uma assistência preventiva.

Sendo assim, percebe-se que a disseminação de informações que estimulem à prevenção e o auxílio às pessoas portadoras de doenças se torna uma ferramenta de extrema importância para aumentar o interesse pela procura da assistência dos serviços 
proporcionados pela ESF. Além disso, é (interessante) fundamental a capacitação do enfermeiro que está à frente da ESF, buscando subsidiá-lo e atualizá-lo nas questões relativas à saúde do homem, para realizar uma assistência eficiente à população masculina.

Desse modo, o presente estudo tem como objetivo identificar na literatura, por meio de uma revisão integrativa, a assistência de enfermagem frente à saúde do homem com foco na execução da Política Nacional de Atenção Integral à Saúde do Homem.

\section{METODOLOGIA}

Este estudo é de caráter bibliográfico com o intuito de agregar conhecimento acerca do tema em debate. Onde se busca por meio de uma revisão integrativa, método que está no âmbito da Prática Baseada em Evidências.

Roman and Friedlander(1998), relatam que a revisão integrativa de pesquisa, possibilita ao interessado reconhecer os profissionais que mais investigam um assunto, suas áreas de atuação e suas contribuições mais relevantes; permite separar o achado científico de opiniões e ideias; permite descrever o conhecimento no seu estado atual; e promove o impacto da pesquisa sobre a prática profissional. Este método permite fazer generalizações sobre determinados assuntos estudados por vários pesquisadores, em diferentes lugares e momentos, mantendo os interessados atualizados e facilitando as modificações da prática cotidiana como consequência da pesquisa.

O processo de elaboração da revisão integrativa encontra-se bem definido na literatura, entretanto, diferentes autores adotam formas distintas de subdivisão de tal processo, com pequenas modificações. No geral, para a construção da revisão integrativa, é preciso percorrer seis etapas distintas: identificação do tema e seleção da questão norteadora da pesquisa; estabelecimento dos critérios de inclusão e exclusão; identificação dos estudos pré-selecionados e selecionados; categorização dos estudos selecionados; análise e interpretação dos resultados e apresentação da síntese da revisão.

Realizou-se um levantamento bibliográfico no banco de dados da Biblioteca Virtual em Saúde (BVS) utilizando, como fontes, a base de dados Literatura Latino- 
Americana e do Caribe em Ciências da Saúde (LILACS), a Base de Dados da Enfermagem (BDENF). A consulta atendeu ao critério da presença dos Descritores em Ciências da Saúde (DeCS): 'Assistência de enfermagem', 'Atenção Primária à Saúde’, 'Masculinidade', 'Política de Saúde', e 'Saúde do Homem'.

Este estudo baseia-se na pesquisa de artigos publicados em periódicos científicos. Foram utilizados os seguintes critérios: na sequência abaixo:

- Disponibilidade do artigo na íntegra;

- Publicados em português;

- Publicados no período de 2014 a 2018;

- Seleção do título que contenha referência aos descritores;

- Leitura classificatória do resumo e leitura do texto na íntegra (também classificatória);

- Utilização de filtros específicos com relação ao tema em questão no momento (na hora) da pesquisa.

Utilizando os descritores citados, foram encontrados um total de1.637 artigos, foram selecionados 50 artigos a partir da leitura de Título e Resumo. Destes, 15 foram escolhidos para ser lidos na íntegra e verificar se tinha relevância com o tema, sendo selecionados 10 artigos para fazer parte desta revisão integrativa.

A coleta de dados foi instrumentalizada através de um quadro onde separamos uma ficha para cada fonte pesquisada, nesse fichamento foram evidenciadas as informações referentes a cada autor como: Ano, Autores, Fonte e Título. Após a coleta desses dados, os autores obtiveram informações que subsidiaram a criação dos resultados da pesquisa, a qual foi realizada através do debate dos autores por meio dos quadros fundamentados pelos resultados encontrados.

\section{HISTÓRIA DO HOMEM NA SAÚDE}

Nota-se que Os estudos sobre homem e saúde começaram a surgir por volta do ano de 1970 nos Estados Unidos. Segundo os estudos sobre a saúde do homem percebeu-se estar em grande desvantagem em comparação à saúde da mulher, pois havia um grande número de mortes de homens. Mais tarde nos anos de 1990, começou a 
se ter mais foco nas singularidades de homens no processo saúde-doença (SCHWARZ, et al., 2012).

De acordo com Schraiber, et al., 2005, estudos sobre homem na saúde passou a ser visto em outras perspectivas. Passou-se a discutir, entre outras coisas, a singularidade do "saudável" e do "doente" entre segmentos masculinos. Isso veio trazendo um novo significado do masculino para buscar-se uma saúde de forma integral ao homem.

Seguindo essa linha de pensamento, surgiram estudos importantes, antes concentradas na saúde da mulher. Começaram a incluir como objeto de estudo o homem, porém, dentro desse contexto, muitos pesquisadores têm interesse nos processos de morbi-mortalidade dos homens, tendo em vista que a masculinidade hegemônica pode trazer riscos à saúde, gerando assim o adoecimento do homem (RODRIGUES E RIBEIRO, 2012).

Keijzer (2003 apud Schraiber, et al., 2005) observou que há na América Latina uma quantidade crescente de programas que se voltam para a experiência de homens em relação à sexualidade, reprodução, paternidade, violência ou a própria saúde. $\mathrm{O}$ autor considera que a inclusão da temática "homens e saúde" poderá ter como resultado a participação dos homens no alcance dos objetivos programáticos dessas organizações, deslocando-se da visão tradicional do homem apenas como parte dos problemas para concebê-lomas também como parte da solução.

Após a instituição da Política Nacional de Atenção Integral à Saúde do Homem (PNAISH), o Brasil passaria ser o primeiro país das américas a ter uma política voltada para a saúde do homem, sendo o segundo no mundo, após a Irlanda ter formulado a sua própria política. No entanto, somente depois de 2008 os homens passaram a ser foco de ações em saúde junto com grupos específicos mais antigos; adolescentes, mulheres, idosos, indivíduos institucionalizados, pessoas com deficiência, etc. (COELHO, et al., 2018).

Nesse sentido, essa política veio para auxiliar os gestores estaduais e municipais dentro do contexto que criou esse Plano de Ação Nacional. A PNAISH veio para destacar a singularidade masculina nos seus diversos contextos socioculturais e 
político-econômicos, bem como aponta princípios para o aumento da expectativa de vida e a redução dos índices de morbimortalidade por causas que possam ser prevenidas e evitadas na população masculina.

\section{MASCULINIDADE E SAÚDE}

A trajetória envolvendo a saúde do homem é um assunto recente e têm acarretado sérios problemas relacionados a esse marco. Nos dias atuais estão apresentando destaque, baseados em uma atenção para sensibilizar o público masculino quanto à sua importância no quesito prevenção em saúde.

O diagnóstico sobre a saúde dos homens aponta duas perspectivas que refletem duplo movimento, pois os determinantes socioculturais de saúde masculina se apresentam como uma barreira pelo fato de que o homem ideal é viril, forte e invulnerável. Tais fenômenos estão ligados a uma questão de gênero. Outro fator, que incide na baixa procura dos homens pelo serviço da unidade básica de saúde é a falta de orientação desses homens, falta de estratégias por meio das equipes para trazer essa população para os atendimentos de rotina (GOMES; NASCIMENTO; ARAUJO, 2007).

Em relação à masculinidade destaca-se a prevenção do câncer de próstata, é um tema a ser apontado para sintetizar o assunto: preconceitos e medos relacionados ao toque retal, sendo um dos problemas no quesito masculinidade.

De acordo com Castro, et al., (2008), os exames de prevenção do câncer de próstata, embora sejam ofertados gratuitamente na rede pública de saúde, tem baixa demanda devido aos obstáculos construídos pelo próprio homem que colocam em evidência o fator masculinidade.

Diante disto, as condutas do homem, por conta dessa concepção estereotipada vêm de certa forma interferir no cuidado e prática de saúde, favorecendo fatores de riscos e adoecimento em várias idades. Percebe-se que, buscando um melhor entendimento das palavras sexo e gênero, compreendemos a verdadeira necessidade de uma análise mais crítica voltada ao tema em estudo, principalmente quando se relaciona o homem com as estruturas do processo saúde doença (BRASIL, 2008). 
Numa perspectiva de consolidação e superação das dificuldades do SUS, foi definido em 2006 o pacto pela saúde, firmado pelo Ministério da Saúde (MS), com os respectivos gestores de saúde. Nesse momento foram estabelecidas três dimensões de atuação: Pacto em defesa do SUS, Pacto pela vida e Pacto de gestão. Dentre estes, o Pacto pela vida assumiu como uma de suas prioridades para aquele ano, o fortalecimento da $\mathrm{AB}$ (Atenção Básica), através da consolidação e qualificação da ESF como modelo da $\mathrm{AB}$ à saúde como centro ordenador das redes de atenção à saúde do SUS. Através do referido pacto, várias políticas foram e estão sendo implementadas na busca de assistência à saúde integral e universal. (BRASIL, 2011).

Diante disso e todas as abordagens atuais, a história das políticas de saúde voltadas a "populações específicas", pode-se considerar a recente criação, pelo Ministério da Saúde, o programa saúde do homem. Nesse contexto, ele evidencia um momento significativo nas atualizações na saúde. Como incentivo desse desenvolvimento, a PNAISH vem sendo um programa satisfatório avaliado positivamente, pois tem como objetivo facilitar e ampliar o acesso com qualidade da população masculina. Com isso, o Ministério da Saúde pretende através da Política Nacional de Saúde do Homem romper os fatores impeditivos, para que os homens frequentem os serviços de saúde e incentivá-los a procurarem a atenção primária a saúde já que na maioria das vezes recorrem aos serviços de saúde apenas quando a doença está instalada ou em estágios mais avançada.

Quando a PNAISH foi instituída, a mesma veio alinhada a PNAB, privilegiando desta forma a ESF, num consenso de evitar a setorização de serviços ou a segmentação de estruturas. Sob esta ótica, a PNAB tem na Saúde da Família sua estratégia prioritária para expansão e consolidação da atenção básica. A qualificação da ESF e de outras estratégias de organização da atenção básica deve seguir as diretrizes da atenção básica e do SUS, configurando um processo progressivo e singular que considera e inclui as especificidades locorregionais (BRASIL, 2012). 
Contudo na elaboração da Política Nacional de Atenção Integral à Saúde do Homem criou uma integração à outras Políticas de Saúde. Desse modo, com essa interação transversal também se reflete na interdependência e necessária cooperação de todos, somando experiências e discutindo exaustivamente diretrizes que possibilitem as melhores opções de construção e operacionalização dessa política. Assim, dará a cada segmento, gestor ou executor, a corresponsabilidade pela correta implementação das ações, em benefício da população a ser assistida (BRASIL, 2009).

Neste sentido a PNAISH afirma princípios consonantes aos do SUS, relacionados por exemplo, à "humanização, qualidade de vida e promoção da integralidade do cuidado na população masculina promovendo o reconhecimento e respeito à ética e aos direitos do homem, obedecendo às suas peculiaridades socioculturais" (BRASIL, 2009).

Sendo assim, a PNAISH apresenta como área de atenção a população masculina de 20 a 59 anos, tem como objetivo melhorar as condições de saúde dos homens e reduzir a morbimortalidade dessa população através de ações de promoção, prevenção e proteção básica à saúde, facilitando o acesso aos serviços de saúde, qualificando e humanizando a atenção integral à saúde do homem. Diante disso, é importante destacar que a PNAISH veio despertar e sensibilizar, tanto os homens quanto os profissionais da saúde, sobre a importância do cuidado da saúde do homem, buscando uma melhor compreensão do processo saúde-doença masculino e o fortalecimento da Atenção Primária (BRASIL, 2009).

Contudo, certamente, a Política Nacional de Atenção Integral à Saúde do Homem veio para colaborar com a saúde do "ser masculino", pois, de alguma forma, as crenças e valores constituídos ao longo dos anos, pelas barreiras socioculturais e também pela resistência masculina à medicina de uma forma geral, estão mudando em relação a sua própria masculinidade, levando o homem a ser mais consciente quanto a sua saúde e a importância da prevenção.

\section{ASSISTÊNCIA DA ENFERMAGEM NA SAÚDE DO HOMEM}


A enfermagem está à frente das ações de promoção à saúde nos serviços de atenção básica o que constituem uma ferramenta importante para atenção à saúde masculina, sendo a participação e o engajamento dos profissionais imprescindíveis para a qualidade das ações.

Nesse sentido podemos conceituar o termo "promoção em saúde" como uma forma de proporcionar às pessoas um maior controle sobre sua saúde, baseando-se principalmente nos princípios de justiça social e equidade. Onde podemos dizer que a educação em saúde se divide em três áreas básicas:

- Promoção da saúde;

- Prevenção (fatores de risco e medidas preventivas);

- Rastreio e diagnóstico precoce.

Branco (2005) fala que os enfermeiros devem assumir no seu cotidiano de prática assistencial, esta sua função, não como uma área complementar, mas integrada no cuidar em enfermagem, onde a educação em saúde constitua uma atividade de igual dimensão a qualquer outra realizada. Bezerra and Júnior (2014) afirmam que "A atuação do enfermeiro na saúde do homem assume um caráter amplo, visto que esse profissional tem seu campo de atuação em ações de promoção, prevenção, recuperação e reabilitação em saúde".

De acordo com Branco (2005), o profissional da saúde como o elemento da equipe de saúde que mais tempo fica em contato com o cliente acaba tendo o papel fundamental na promoção da saúde e na prevenção das doenças e outros agravos à saúde. A sua função deve privilegiara educação em saúde, a aquisição de hábitos saudáveis, a descoberta de novas motivações e de outros fatores determinantes do comportamento.

Desse modo se o enfermeiro tiver em conta estes aspectos e utilizar estratégias educativas adequadas poderá ajudar os indivíduos a adotar ou a alterar comportamentos que melhorem a sua saúde e previnam problemas como o câncer. 
Nesta revisão integrativa foram coletados e analisados dados referentes aos anos de 2014 a 2018. Os dados que integram o espectro de assuntos relacionado ao tema: saúde do homem. No descritor: assistência de enfermagem foram encontrados 5 artigos; atenção primária à saúde foram encontrados 10 artigos; masculinidade foram encontrados 6 artigos; política de saúde foram encontrados 10 artigos; saúde do homem foram encontrados 19 artigos.

Descritos na tabela 1, através de uma leitura seletiva e minuciosa considerando apenas pesquisas que atendiam aos objetivos, foram selecionados 10 artigos.

Tabela 1: Panorama geral dos artigos avaliados, incluídos nos resultados e discussão.

\begin{tabular}{|c|c|c|c|c|c|}
\hline DESCRITOR & $\mathbf{N}^{\circ}$ & AUTORES/ANO & FONTE & TÍTULO & $\begin{array}{l}\text { DELINEAME } \\
\text { NTO }\end{array}$ \\
\hline \multirow[t]{3}{*}{$\begin{array}{l}\text { Assistência de } \\
\text { Enfermagem }\end{array}$} & 01 & Freitas et al., (2018) & BDENF & $\begin{array}{ll}\text { Práticas } & \text { de } \\
\text { Cuidado } & \text { de } \\
\text { Enfermagem } & \text { no } \\
\text { Contexto } & \text { da } \\
\text { Política } & \text { do } \\
\text { Homem } & \\
\end{array}$ & $\begin{array}{l}\text { Quantitativo } \\
\text { Descritivo }\end{array}$ \\
\hline & 02 & Ribeiro et al., (2014) & BDENF & $\begin{array}{ll}\text { Saúde } & \text { dos } \\
\text { Homens: } & \\
\text { Abordagem } & \text { na } \\
\text { Formação } & \text { de } \\
\text { Enfermeiros } & \end{array}$ & $\begin{array}{l}\text { Documental } \\
\text { Qualitativa }\end{array}$ \\
\hline & 03 & Cavalcanti et & LILACS & Assistência & Descritivo \\
\hline
\end{tabular}




\begin{tabular}{|c|c|c|c|c|c|}
\hline \multirow[b]{2}{*}{$\begin{array}{l}\text { Atenção primária } \\
\text { à saúde }\end{array}$} & & (2014) & & $\begin{array}{l}\text { Integral a Saúde } \\
\text { do Homem: } \\
\text { Necessidades, } \\
\text { Obstáculos e } \\
\text { Estratégias de } \\
\text { Enfrentamento }\end{array}$ & $\begin{array}{l}\text { exploratório, } \\
\text { com abordagem } \\
\text { qualitativa }\end{array}$ \\
\hline & 04 & Lemos et al., (2017) & BDENF & \begin{tabular}{lr} 
Saúde & do \\
Homem: & os \\
Motivos & da \\
Procura & dos \\
Homens & pelos \\
\multicolumn{2}{l}{ Serviços de Saúde } \\
\end{tabular} & $\begin{array}{l}\text { Exploratório, de } \\
\text { abordagem } \\
\text { qualitativa }\end{array}$ \\
\hline \multirow[b]{2}{*}{ Masculinidade } & 05 & Teixeira (2016) & LILACS & \begin{tabular}{lr} 
Atenção à Saúde \\
do & Homem: \\
Análise da & sua \\
Resistência & na \\
Procura & dos \\
\multicolumn{2}{l}{ Serviços de Saúde } \\
\end{tabular} & $\begin{array}{l}\text { Descritivo com } \\
\text { abordagem } \\
\text { qualitativa }\end{array}$ \\
\hline & 06 & Sousa et al., (2016) & BDENF & $\begin{array}{l}\text { Homens nos } \\
\text { Serviços de } \\
\text { Atenção Básica à } \\
\text { Saúde: } \\
\text { Repercussões da } \\
\text { Construção Social } \\
\text { das } \\
\text { Masculinidades }\end{array}$ & $\begin{array}{l}\text { Descritivo } \\
\text { Qualitativo }\end{array}$ \\
\hline \multirow[t]{2}{*}{ Política de saúde } & 07 & Assis et al., (2018) & LILACS & $\begin{array}{lr}\text { Atuação } & \text { dos } \\
\text { Enfermeiros } & \\
\text { Frente à } & \text { Política } \\
\text { Nacional } & \text { de } \\
\text { Atenção Integral a } & \text { Saúde } \\
\text { Homem: } & \text { do } \\
\text { Estudo } & \\
\text { Exploratório } & \\
\end{array}$ & $\begin{array}{l}\text { Descritiva- } \\
\text { exploratória } \\
\text { com abordagem } \\
\text { qualitativa }\end{array}$ \\
\hline & 08 & Moura et al., (2014) & LILACS & $\begin{array}{lr}\text { Atenção à Saúde } \\
\text { dos Homens } & \text { no } \\
\text { Âmbito } & \text { da } \\
\text { Estratégia } & \text { Saúde } \\
\text { da Família } & \\
\end{array}$ & Quantitativo \\
\hline \multirow[b]{2}{*}{ Saúde do homem } & 09 & Santos e Santos (2017) & LILACS & $\begin{array}{l}\text { Onde estão os } \\
\text { Homens? O que } \\
\text { os Distanciam ou } \\
\text { os Aproximam } \\
\text { dos Serviços da } \\
\text { Atenção Primária } \\
\text { à Saúde }\end{array}$ & $\begin{array}{ll}\text { Qualitativo } & \text { de } \\
\text { natureza } & \\
\text { exploratória } & \text { e } \\
\text { descritiva } & \end{array}$ \\
\hline & 10 & Araújo et al., (2014) & BDENF & $\begin{array}{lr}\text { Saúde } & \text { do } \\
\text { Homem: } & \text { Ações e } \\
\text { Serviços } & \text { na } \\
\text { Estratégia } & \text { Saúde } \\
\text { da Família } & \end{array}$ & $\begin{array}{l}\text { Exploratório e } \\
\text { descritivo com } \\
\text { abordagem } \\
\text { qualitativa }\end{array}$ \\
\hline
\end{tabular}

Fonte: Autores (2019). 
Freitas, et al., (2018), através de um estudo qualitativo descritivo, dizem que: As ações da política estão contempladas, parcialmente, no cotidiano dos profissionais de Enfermagem na instituição hospitalar e se aproximam do contexto da política do homem demonstrada pelo envolvimento profissional, a integralidade dos cuidados, o acolhimento, a humanização do cuidado ao homem e a qualidade da assistência como, também, a dificuldade de implementar integralmente a política na prática.

Ribeiro, et al., (2014), relatam que o estudo da saúde dos homens é recente, no meio acadêmico, e implica considerá-los em todas as etapas da vida, no contexto social, familiar, bem como no direito a ter a atenção à saúde de maneira integral e humanizada. Dessa maneira, este estudo aponta, aos Cursos de Graduação em Enfermagem, a necessidade de reelaborar em seus currículos e inserir temas de atenção específica à saúde dos homens, abordando questões socioculturais que se inter-relacionam com a prevenção de doenças e agravos à saúde da população masculina.

A pesquisa de Cavalcanti et al., (2014), propiciou a oportunidade de ouvir os homens durante as entrevistas e compreender um pouco do universo masculino, a partir do olhar dos mesmos sobre a saúde através das suas necessidades, dos obstáculos encontrados nos serviços de saúde e as estratégias de enfrentamento as barreiras elencadas. O pressuposto que norteou este estudo foi ratificado com destaque para as necessidades de prevenção de doenças e promoção da saúde do homem, o que representa um avanço diante da superioridade masculina, característica, geralmente, machista que associa o homem a um ser invulnerável.

Sucessivamente, Lemos et al., (2017), sugerem a necessidade de ampliar as discussões sobre a temática de atenção à saúde do homem, considerando as especificidades e complexidades do universo do ser masculino. Isto pois as temáticas relacionadas à saúde do homem e à masculinidade, bem como as práticas de prevenção e promoção da saúde, não são abordadas de forma adequada nem no âmbito da saúde e nem da educação, e isto tem contribuído para os impasses e resistências associados ao atendimento da população masculina. 
Teixeira e Cruz (2016) evidenciaram que os homens são resistentes no cuidado da sua saúde devido aos sentimentos de medo, vergonha, e por causas comportamentais como a impaciência, o descuido, prioridades de vida, e ainda com questões relacionadas com a forma de organização dos serviços de saúde. Observou-se que os fatores ligados ao gênero exercem forte influência, muitas vezes até como obstáculo.

Sousa et al., (2016) cita que é necessário intensificar as ações de promoção, atenção e vigilância, que estimulem neles a procura aos serviços, poderá ser um caminho para reduzir os altos índices de hospitalização, adoecimento, violência, uso abusivo do álcool e outras drogas, acidentes, cânceres e outras morbimortalidades que se transformam em prejuízos irreparáveis para o indivíduo, a família e a sociedade, além de gerar maiores custos ao Sistema Único de Saúde.

O estudo realizado por Assis, et al., (2018), permitiu verificar a importância do enfermeiro como elemento essencial na atenção primária. Entre suas atribuições, destacam-se as ações de promoção da saúde voltada para população masculina, foco do estudo, e que estejam em consonância com a PNAISH.

Moura, et al., (2014) constatou que embora haja interesse dos homens em ampliar sua participação nas atividades desenvolvidas pelas equipes das ESF/UBS, uma vez que dirigidas às suas reais necessidades, ainda há lacunas significativas a serem preenchidas, desde a adequação da estrutura/ambiência para o atendimento na atenção básica à motivação e o desenvolvimento de ações de promoção, tratamento e recuperação dos agravos mais frequentes nesta população (causas externas, por exemplo).

Santos e Santos (2017) descreveram que à Saúde do Homem ainda há muito a ser feito para atrair este público a estar mais presente na rede de serviços do SUS. Envolvem desde medidas estruturais e organizacionais dos serviços da Atenção Básica, até mesmo ações que visam estimular os homens para o cuidado com a sua saúde, no sentido de corresponsabilizá-los pela sua própria saúde para que seja motivado para a busca dos serviços de saúde.

Por fim, o estudo exploratório e descritivo com abordagem qualitativa de Araújo et al (2014), propuseram que mecanismos de promoção à saúde sejam inseridos 
nas práticas cotidianas dos profissionais, a partir de cuja capacitação a PNAISH possa ser executada, além de sensibilização da gestão de saúde, fazendo-a compreender as necessidades de saúde do homem, possibilitando assim a melhoria do acesso e da saúde da população masculina.

\section{CONCLUSÃO}

Este estudo, realizado através de uma revisão integrativa evidencia que, a PNAISH vem construindo um cenário para a discussão da saúde masculina e para a implementação de recursos de saúde que visam fortalecer a prevenção e promoção da saúde dos homens. Entretanto, conclui-se que as ações de implementação da política ainda não foram suficientes para inserir efetivamente o público masculino nos serviços de saúde pela via de atenção primária à saúde, chegando estes ainda pelas portas dos serviços emergenciais e das especialidades, e com quadros de saúde já crônicos ou em situações agudas.

Sugere-se investir na difusão da política a partir da capacitação profissional e da maior divulgação pelos meios de comunicação, além do investimento em estratégias que visem a aproximação dos homens aos serviços de saúde, com uma captação precoce e a educação popular, como prevê a política, onde todos os âmbitos da gestão em saúde e também os cenários de formação profissional em saúde devem estimular discussões a respeito da política e buscar maior sensibilização de estudantes, profissionais e da própria comunidade para o tema da atenção integral a saúde do homem.

Seguindo neste sentido, devem-se criar oportunidades e condições para que os homens também possam ser ouvidos e qualificados para criarem seu próprio processo de produção de saúde. Há que se levar em conta as particularidades do público masculino, desde as questões de gênero e cultura, com o intuito de garantir, na saúde, um atendimento qualificado e integral. 
ARAÚJO, M. G, et al. Saúde do homem: ações e serviços na Estratégia Saúde da Família. Rev. enferm. UFPE online 8, no. 2 (2014): 264-271.

ASSIS, N. O, et al. Atuação dos enfermeiros frente à política nacional de atenção integral a saúde do homem: um estudo exploratório. Arquivos de Ciências da Saúde da UNIPAR 22, no. 3 (2018).

BEZERRA, E. A. F, and JÚNIOR J. J. A. O papel do enfermeiro na promoção à saúde do homem: o contexto das unidades básicas de saúde da cidade de macaíba/rn. SANARE-Revista de Políticas Públicas 13.2 (2014).

BRANCO, I. M. B. H. P. Prevenção do câncer e educação em saúde: opiniões e perspectivas de enfermagem. Texto contexto-enferm., Florianópolis 14.2 (2005).

BRASIL. Ministério da Saúde. Secretaria de Atenção à Saúde, Departamento de Ações Programáticas Estratégicas - Política Nacional de Atenção Integral à Saúde do Homem: Princípios e Diretrizes, Brasília, 2008.

BRASIL. Ministério da Saúde. Política Nacional de Atenção Integral à Saúde do Homem. Plano de Ação Nacional (2009-2011). Brasília, 2009.

CASTRO, Luannade, et al. A política nacional de saúde do homem: uma reflexão sobre a questão de gênero. Enfermagem em Foco 2.4 (2011): 215-217.

COELHO, E.B.S et al. Política nacional de atenção integral a saúde do homem. Disponível em: <www.unasus.ufsc.br>. aceso em: 26 nov 2018.

CAVAlCANTI, J. R. D, et al. Assistência Integral a Saúde do Homem: necessidades, obstáculos e estratégias de enfrentamento. Escola Anna Nery Revista de Enfermagem 18, no. 4 (2014): 628-634.

FREITAS, E. R, et al. Práticas de cuidado de enfermagem no contexto da política do homem. (2018).

GOMES, Romeu, NASCIMENTO, E.F and ARAÚJO F.C. Por que os homens buscam menos os serviços de saúde do que as mulheres? As explicações de homens com baixa escolaridade e homens com ensino superior. Cadernos de Saúde Pública 23 (2007): 565-574.

LEMOS, A. P, et al. Saúde do homem: os motivos da procura dos homens pelos serviços de saúde. Rev. enferm. UFPE online 11, no. supl. 11 (2017): 4546-4553.

MAIA, L.F.S. Câncer de próstata: preconceitos, masculinidade e a qualidade de vida. Revista Recien-Revista Científica de Enfermagem 2.6 (2012): 16-20. 
MOURA, E. C,

. et al. Atenção à saúde dos homens no âmbito da Estratégia Saúde da Família. Ciência \& Saúde Coletiva 19 (2014): 429-438.

RIBEIRO, D. B,

. et al. Saúde dos homens: abordagem na formação de enfermeiros [Men'shealth: dealingwith training of nurses]. Revista Enfermagem UERJ 22, no. 4 (2014): 540545 .

RODRIGUES, J.F and RIBEIRO, E.R. O homem e a mudança de pensamento em relação à sua saúde. Caderno Saúde e Desenvolvimento 1.1 (2012): 74-86.

ROMAN, A. R, and FRIEDLANDER M. R. Revisão integrativa de pesquisa aplicada à enfermagem. Cogitare Enfermagem 3.2 (1998).

SANTOS, K. O, and SANTOSE. M. Onde estão os homens? O que os distanciam ou os aproximam dos serviços da atenção primária à saúde. Semina: Ciências Biológicas e da Saúde 38, no. 1 (2017): 79-88.

SCHRAIBER, L.B, GOMES, R.G and COUTO M.T. Homens e saúde na pauta da Saúde Coletiva. Ciência \& Saúde Coletiva 10 (2005): 7-17.

SCHWARZ, Eduardo, et al. Política de saúde do homem. Revista de Saúde Pública 46 (2012): 108-116.

SOUSA, A. R, et al. Homens nos serviços de Atenção Básica à Saúde: repercussões da construção social das masculinidades. Revista Baiana de Enfermagem 30 , no. 3 (2016).

TEIXEIRA, D. B. S. Atenção à saúde do homem: análise da sua resistência na procura dos serviços de saúde. Revista Cubana de Enfermería 32, no. 4 (2016). 